\title{
MÔ HÌNH CƠ SỞ DŨ LIỆU DẠNG KHỐI
}

\author{
NGUYỄN XUÂN HUY(1), TRỊNH ĐİNH THĂNG ${ }^{(2)}$
}

\begin{abstract}
At the present time, some types of database models are considered, in which three types of database models are objects of interest: Hierachiral model, Network model and Relational model. This paper introduces a new model for databases, which called a database model of block form. Some basis concepts and block form - relational algebra are introduced for this model. Some of properties related to keys of block schema and block form - relational algebra are claimed and proved. It is shown that the relational database model is special case of the database model of block form in which each relation is a block with the index set, containing only one element.
\end{abstract}

\section{Mở ĐẦU}

Mô hình cơ sớ dữ liệu quan hệ do Codd E. F. đưa ra [5] là một họ các quan hệ, trong đó các quan hệ có cấu trúc phẳng (tuyến tính). Do cấu trúc phẳng của quan hệ nên mô hình này chưa đủ đáp ứng đối với các ứng dụng phức tạp, các cơ sở dữ liệu có cấu trúc phi tuyến...

Để mở rộng và khắc phục phần nào những nhược điểm của mô hình quan hệ truyền thống nói trên, bài báo này đưa ra một mô hình cơ sớ dữ liệu mới gọi là mô hình cơ sơ dữ liệu dạng khối. Mô hình này giúp biểu diễn thế giới thực trong quá trình vận động một cách tự nhiên hơn.

Theo truyền thống của lý thuyết cơ sở dữ liệu ta sẽ sử dụng các kí hiệu sau đây:

$\begin{array}{ll}\text { Kí hiệu } & \text { Ý nghĩa } \\ A, B, C & \text { thuộc tính } \\ X, Y, Z & \text { tập thuộc tính } \\ X Y & X \cup Y \text { (hợp của } 2 \text { tập thuộc tính } X \text { và } Y \text { ) } \\ A B C & \{A, B, C\} \text { (tập thuộc tính gồm } 3 \text { phần tử } A, B, C \text { ) } \\ \operatorname{dom}(A) & \text { miền giá trị cưa thuộc tính } A\end{array}$

Phần còn lại của bài báo này được chia thành 4 phần. Khái niệm về khối, lát cắt và các mệnh đề liên quan với chúng được trình bày trong phần 2 . Phần 3 gồm khái niệm về khóa cùng một vài tính chất. Các phép tính trên cơ sở dữ liệu dạng khối được đưa ra ơ phần 4 , và cuối cùng, phần 5 trình bày đại số quan hệ dạng khối.

\section{KHỐI VA LÅT CÅT}

Khái niệm toán học cưa mô hình cơ sở dữ liệu dạng khối (gọi tắt là mô hình khối).

Định nghĩa 2.1. Gọi $R=$ (id; $A_{1}, A_{2}, \ldots, A_{n}$ ) là một bộ hữu hạn các phần tứ, trong đó id là tập chỉ số hữu hạn khác rỗng, $A_{i}(i=\overline{1, n})$ là các thuộc tính. Mỗi thuộc tính $A_{i}(i=\overline{1, n})$ 
có miền giá trị tương ứng là $\operatorname{dom}\left(A_{i}\right)$. Một khối $r$ trên $R$, kí hiệu $r(R)$ gồm một số hữu hạn phần tư mà mỗi phần tử là một họ các ánh xạ từ tập chỉ số id đến các miền trị của các thuộc tính $A_{i},(i=\overline{1, n})$. Nói cách khác:

$$
t \in r(R) \Leftrightarrow t=\left\{t^{i}: \mathrm{id} \rightarrow \operatorname{dom}\left(A_{i}\right)\right\}, i=\overline{1, n} .
$$

Ta kí hiệu khối đó là $r(R)$ hoặc $r\left(\mathrm{id} ; A_{1}, A_{2}, \ldots, A_{n}\right)$ đôi khi nếu không sợ nhầm lẫn ta kí hiệu đơn giản là $r$.

Khi đó khối $r(R)$ được gọi là có lược đồ khối $R$. Như vậy trên cùng một lược đồ khối $R$ ta có thể xây dựng được nhiều khối khác nhau.

Ví dụ: Cho khối NS(R), ơ đây $R=\left(\right.$ id; $\left.A_{1}, A_{2}, A_{3}, A_{4}\right)$,

$$
\text { trong đó: } \text { id }=\{1993,1995,1998\}
$$

$$
A_{1}=\text { ma, } A_{2}=\text { ten, } A_{3}=\text { luong, } A_{4}=\text { trinh_do }
$$

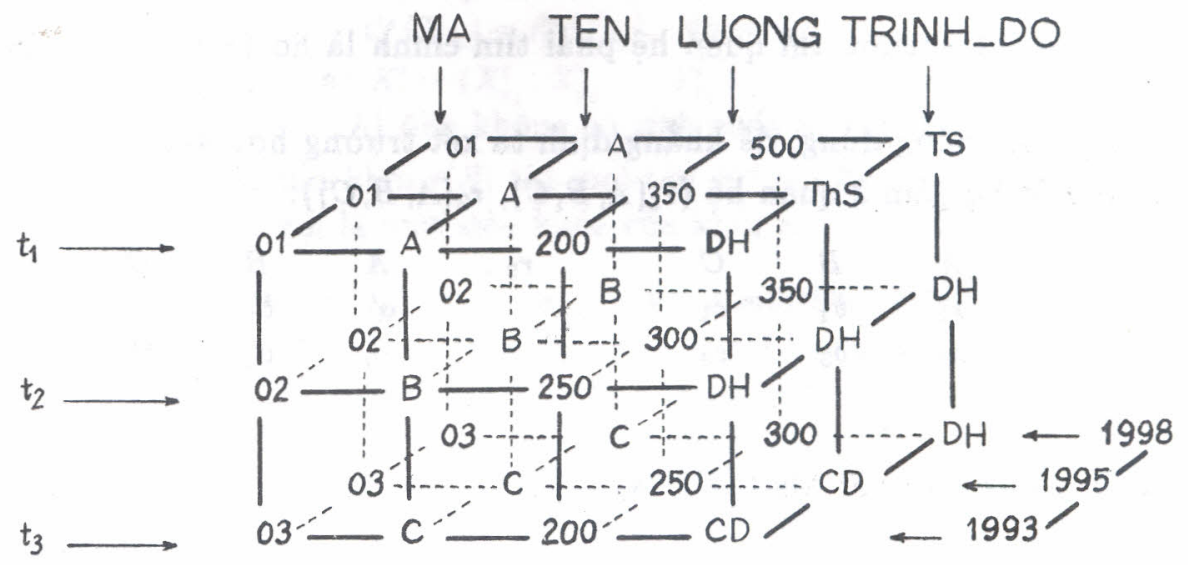

Với khối NS $(R)$ ờ trên ta thấy nó gồm 3 phần tự: $t_{1}, t_{2}, t_{3}$. Khi đó ta có: $t_{1}(1993$, luong $)=$ $200, t_{2}(1995$, luong $)=300, t_{3}(1998, \mathrm{ma})=03, t_{3}(1995$, trinh_do $)=$ 'CD' $\ldots$

Định nghĩa 2.2. Cho $R=\left(\mathrm{id} ; A_{1}, A_{2}, \ldots, A_{n}\right), r(R)$ là một khối trên $R$. Vói mối $x \in$ id ta kí hiệu $r\left(R_{x}\right)$ là một khối với $R_{x}=\left(\{x\} ; A_{1}, A_{2}, \ldots, A_{n}\right\}$ sao cho:

$$
t_{x} \in r\left(R_{x}\right) \Leftrightarrow t_{x}=\left\{t_{x}^{i}=\left.t^{i}\right|_{x}\right\}_{i=\overline{1, n}} \text { với } t \in r(R), \text { và } t=\left\{t^{i}: \operatorname{id} \rightarrow \operatorname{dom}\left(A_{i}\right)\right\}_{i=\overline{1, n}} .
$$

O’’ đây $t_{x}^{i}(x)=t^{i}(x)$ với $i=\overline{1, n}$.

Khi đó, $r\left(R_{x}\right)$ được gọi là một lát cắt trên khối $r(R)$ tại điểm $x$.

Ví dụ: Vói khối NS(R) đã cho ờ trên, $R=\left(\mathrm{id} ; A_{1}, A_{2}, A_{3}, A_{4}\right)$, trong đó: $\mathrm{id}=\{1993,1995,1998\}$

$$
A_{1}=\text { ma, } A_{2}=\text { ten, } A_{3}=\text { luong, } A_{4}=\text { trinh_do }
$$

Nếu $x=1995 \in$ id thì lát cắt $r\left(R_{1995}\right)$ có dạng như sau:

$\begin{array}{cccc}\text { MA } & \text { TEN } & \text { LUONG } & \text { TRINH_DO } \\ 01 & A & 350 & \text { ThS } \\ 02 & B & 300 & \text { DH } \\ 03 & C & 250 & \text { CD }\end{array}$


Ta có các mệnh đề sau.

Mệnh đề 2.1. Cho $R=\left(i d ; A_{1}, A_{2}, \ldots, A_{n}\right), r(R)$ là một khối trên $R$. Với mỗi $x \in$ id thi lât căt $r\left(R_{x}\right)$ là một quan. hệ. Trong trường hợp tập chí số id chí gồm một phần tử thì $r(R)$ trơ thành một quan hệ.

Như vậy mỗi quan hệ $r\left(A_{1}, A_{2}, \ldots, A_{n}\right)$ là một trường hợp đặc biệt cưa khối, đó chính là khối $r(R)$ với $R=\left(\{x\} ; A_{1}, A_{2}, \ldots, A_{n}\right)$.

Mệnh đề 2.2. Cho $R=\left(i d ; A_{1}, A_{2}, \ldots, A_{n}\right), r(R)$ ld̀ một khối trên $R$. Khi đó tồn tại một họ quan hệ duy nhất biểu diễn họ $\left\{r\left(R_{x}\right)\right\}_{x \in \text { id }}$ các lát căt cuia khối $r(R)$. Ngrợc lại không đưng, nghĩa là với một họ quan hệ cho trước biểu diễn họ các lát cåt cuia một khối nào đó thi khối tim dược khồng duy nhất.

\section{Chưng minh:}

1) Với khối $r(R)$ cho trước thì quan hệ phái tìm chính là họ $\left.\left\{r(R)_{x}\right)\right\}_{x \in \text { id }}$ các lát cắt cưa khối $r(R)$.

2) Điều ngược lại không đúng, để khẳng định ta xét trường hợp sau:

Giả sử ta có một họ gồm 2 quan hệ $\left\{r_{1}(A, B, C), r_{2}(A, B, C)\right\}$ :

$\begin{array}{cccccccc}r_{1}: & A & B & C & r_{2}: & A & B & C \\ & a_{1} & b_{1} & c_{1} & & a_{1}^{\prime} & b_{1}^{\prime} & c_{1}^{\prime} \\ & a_{3} & b_{3} & c_{3} & & a_{2}^{\prime} & b_{2}^{\prime} & c_{2}^{\prime} \\ & & & & a_{3}^{\prime} & b_{3}^{\prime} & c_{3}^{\prime}\end{array}$

Khi đó ta có chằng hạn các khối sau đây nhận họ hai quan hệ $\left\{r_{1}, r_{2}\right\}$ nói trên là họ các lát cắt của nó:

$r(R)$

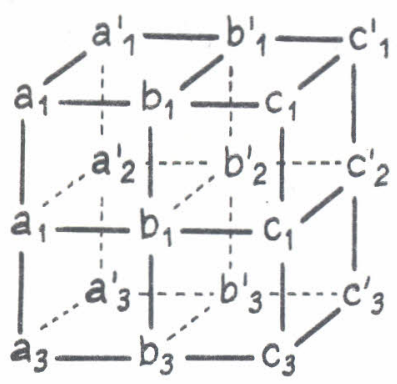

$S(R)$

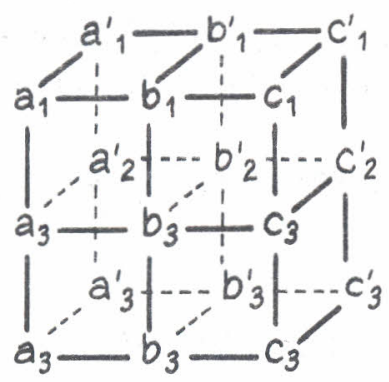

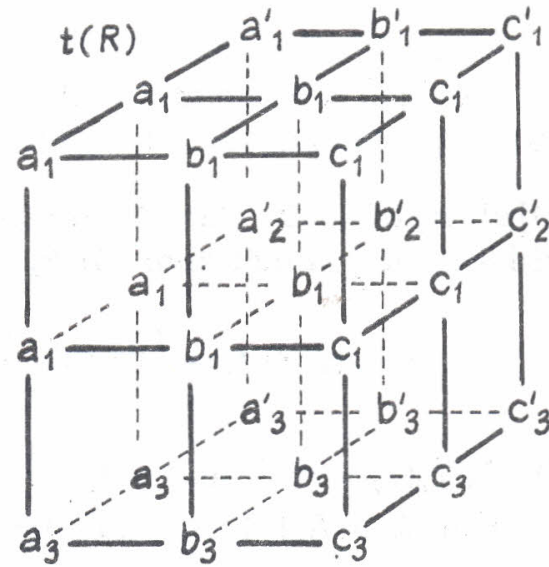

Như vậy sự tồn tại của các khối có họ các lát cắt là họ quan hệ $\left\{r_{1}, r_{2}\right\}$ nói trên là không duy nhất.

\section{KHÓA CỮA KHỐI $r(R)$}

Cho $R=$ (id; $\left.A_{1}, A_{2}, \ldots, A_{n}\right), r$ là một khới trên $R$. Với mỗi $x \in \mathrm{id}, t \in r(R), t=$ $\left(t^{1}, t^{2}, \ldots, t^{n}\right)$, ta kí hiệu $t\left(x ; A_{i}\right)(i=1, . ., n)$ là giá trị của phần tứ $t$ ớ thuộc tính $A_{i}$ tại chí số $x$. 
Để thuận lợi cho việc trình bày, ta đặt $x_{i}=\left(x ; A_{i}\right), x \in$ id và như vậy:

$t\left(x_{i}\right)=t\left(x ; A_{i}\right)=t^{i}(x)(i=1, \ldots, n)$. Từ đó, ta kí hiệu:

$$
\mathrm{id}_{i}=\left\{x_{i}\right\}_{x \in \mathrm{id}} \text {, như vậy } \mathrm{id}_{i}=\left\{\left(x ; A_{i}\right)\right\}_{x \in \mathrm{id}} .
$$

Với $X_{i} \subseteq$ id $_{i}$ thì ta kí hiệu: $t\left(X_{i}\right)=\left\{t\left(y_{i}\right) \mid y_{i} \in X_{i}\right\}$.

Giá sư $t_{1}, t_{2} \in r(R)$ với $t_{1}=\left\{t_{1}^{i}: \mathrm{id} \rightarrow \operatorname{dom}\left(A_{i}\right)\right\}_{i=1, \ldots, n}, t_{2}=\left\{t_{2}^{i}: \mathrm{id} \rightarrow \operatorname{dom}\left(A_{i}\right)\right\}_{i=1, \ldots, n}, \mathrm{khi}$ đó ta định nghĩa khóa của khối $r(R)$ như sau:

Định nghĩa 3.1. Khóa của khối $r$ trên lược đồ khối $R=\left(\mathrm{id} ; A_{1}, A_{2}, \ldots, A_{n}\right.$ ) là một tập $K=\left\{X_{i_{1}}, X_{i_{2}}, \ldots, X_{i_{h}}\right\}$, trong đó $X_{i_{k}} \neq \emptyset, X_{i_{k}} \subseteq \mathrm{id}_{i_{k}}(k=1, \ldots, h)$, thóa mãn 2 tính chất:

a) Với bất kỳ 2 phần tư $t_{1}, t_{2} \in r$ đều tồn tại một $X_{i_{k}} \in K$ sao cho:

$$
t_{1}^{i_{k}}\left(X_{i_{k}}\right) \neq t_{2}^{i_{k}}\left(X_{i_{k}}\right) \text {. }
$$

Nói cách khác, không tồn tại 2 phần từ mà:

$$
t_{1}^{i_{k}}\left(X_{i_{k}}\right)=t_{2}^{i_{k}}\left(X_{i_{k}}\right), \forall k=1, \ldots, h \text {. }
$$

b) Vói bất kỳ tập $K^{\prime}$ nào $K^{\prime}=\left\{X_{i_{1}}^{\prime}, X_{i_{2}}^{\prime}, \ldots, X_{i_{h}}^{\prime}\right\}$, với $X_{i_{k}}^{\prime} \subseteq X_{i_{k}},(k=1, \ldots, h)$ và tồn tại $X_{i_{m}}^{\prime} \subset X_{i_{m}}$, với $m \in\{1,2, \ldots, h\}$ đều không có tính chất a) nói trên.

Nếu tập $K$ là khóa của khối $r(R)$ thì mọi tập $K^{\prime \prime}=\left\{X_{i_{1}}^{\prime \prime}, X_{i_{2}}^{\prime \prime}, \ldots, X_{i_{n}}^{\prime \prime}\right\}$, trong đó $X_{i_{k}} \subseteq$ $X_{i_{k}}^{\prime \prime}(\forall k=1, \ldots, h)$, được gọi là một siêu khóa của khối $r$.

Mệnh đề 3.1. Cho $R=\left(i d ; A_{1}, A_{2}, \ldots, A_{n}\right), r(R)$ là một khối trên $R$, id $=\{x\}$. Khi đó $r(R)$ trở thành quan hệ $r\left(A_{1}, A_{2}, \ldots, A_{n}\right)$ và mối khóa $K=\left\{X_{i_{1}}, X_{i 2}, \ldots, X_{i_{h}}\right\}$ trong đóo $X_{i_{k}} \subseteq i d_{i_{k}}$ $(k=1, \ldots, h)$ cúa khối $r(R)$ lại trơ thành khóa của quan hệ $r\left(A_{1}, A_{2}, \ldots, A_{n}\right)$.

Mệnh đề 3.2. Cho $R=\left(i d ; A_{1}, A_{2}, \ldots, A_{n}\right), r(R)$ là một khối trên $R$, khi đó với $x \in i d$ mà ta có $\left\{X_{i_{1}}, X_{i_{2}}, \ldots, X_{i k}\right\}$ là khóa khối $r(R)$ thi ta cũng có với mọi $y \in i d,\left\{A_{i_{1}}, A_{i_{2}}, \ldots, A_{i_{k}}\right\}$ là khóa của quan hệr $r\left(R_{y}\right)$.

Mệnh đề 3.3. Cho $R=\left(i d ; A_{1}, A_{2}, \ldots, A_{n}\right), r(R)$ là một khối trên $R$. Khi đó nếu với $x \in i d$ nào đó mà ta có $\left\{X_{i_{1}}, X_{i_{2}}, \ldots, X_{i_{k}}\right\}$ là khóa cúa lát cắt $r\left(R_{x}\right)$ thi $\left\{i d_{i_{1}}, i d_{i_{2}}, \ldots, i d_{i_{k}}\right\}$ là khóa của khối $r(R)$.

\section{CÁC PHÉP TÍNH TRÊN CO' SƠ DŨ LỊ̂U DẠNG KHỐI}

Các phép tính cơ bán thường được áp dụng cho một cơ sở dữ liệu là:

- phép chèn (insert)

- phép loại bó (delete)

- phép cập nhật (change)

Trong mô hình cơ sớ dữ liệu dạng khối thì các phép tính này cũng được áp dụng cho từng phần tứ của các khối lưu trữ trong máy. Cụ thể như sau:

a) Phép chèn: Khi chèn thêm một phần tử $t$ vào khối $r$ ta có:

$r=r \cup t$

INSERT $\left(r ; t^{1}, t^{2}, \ldots, t^{n}\right)$

b) Phép loại bo: Phép loại bó là phép xóa một phần tử ra khói một khối cho trước. Chăng hạn, phép loại bó phần tử $t_{0}$ ra khỏi khối $r$ có dạng:

$$
\begin{aligned}
& r=r-t_{0} \\
& \operatorname{DEL}\left(r ; t^{1}=t_{0}^{1}, t^{2}=t_{0}^{2}, \ldots, t^{n}=t_{0}^{n}\right)
\end{aligned}
$$


c) Phép cập nhật: Cũng giống như phép chèn và phép loại bó, phép cập nhật phần tử to thành phần tứ $t_{0}^{\prime}$ có dạng:

$$
\begin{aligned}
& r=r-t_{0} \cup t_{0}^{\prime} \\
& \mathrm{CH}\left(r ; t^{1}=t_{0}^{1}, t^{2}=t_{0}^{2}, \ldots, t^{n}=t_{0}^{n} ; t^{i_{1}}=t^{i_{1}{ }^{\prime}}, t^{i_{2}}=t^{i_{2}{ }^{\prime}}, \ldots, t^{i_{h}}=t^{i{ }^{\prime}}\right)
\end{aligned}
$$

Đối với phép loại bó và phép cập nhật, để xác định một phần tử cần loại bỏ hay cập nhật thì thay vì ta phải dùng bộ ánh xạ $\left(t^{1}, t^{2}, \ldots, t^{n}\right)$ để xác định phần tử $t=\left(t^{1}, t^{2}, \ldots, t^{n}\right)$ như ở trên, ta có thể chỉ dùng các ánh xạ thu gọn mà miền xác định cưa chúng tạo nên khóa cưa khối $r(R)$.

Chằng hạn, nếu khóa của khối $r(R)$ là bộ $K=\left(X_{m_{1}}, X_{m_{2}}, \ldots, X_{m_{k}}\right)$ thì phép loại bó phần tử t $t_{0}$ cửa khối $r$ có dạng:

$$
r=r-t_{0} \text {, }
$$

$\operatorname{DEL}\left(r ; t^{m_{1}}\left(X_{m_{1}}\right)=t_{0}^{m_{1}}\left(X_{m_{1}}\right), t^{m_{2}}\left(X_{m_{2}}\right)=t_{0}^{m_{2}}\left(X_{m_{3}}\right), \ldots, t^{m_{k}}\left(X_{m_{k}}\right)=t_{0}^{m_{k}}\left(X_{m_{k}}\right)\right)$ và phép cập nhật phần tứ $t_{0}$ thành phần từ $t_{0}^{n}$ có dạng

$$
\begin{aligned}
& r=r-t_{0} \cup t_{0}^{\prime}, \\
& \mathrm{CH}\left(r ; t^{m_{1}}\left(X_{m_{1}}\right)=t_{0}^{m_{1}}\left(X_{m_{1}}, t^{m_{2}}\left(X_{m_{3}}\right)=t_{0}^{m_{2}}\left(X_{m_{2}}, \ldots, t^{m_{k}}\left(X_{m_{k}}\right)=t_{0}^{m_{k}}\left(X_{m_{k}}\right)\right.\right.\right. \\
& \left.t^{i_{1}}=t^{i_{1}{ }^{\prime}}, t^{i_{2}}=t^{i^{2}{ }^{\prime}}, \ldots, t^{i_{h}}=t^{i_{h}}\right) .
\end{aligned}
$$

Đối với các phép chèn, loại bỏ và cập nhật nêu trên thì khối $r$ suy biến thành một quan hệ, nghĩa là khi tập chỉ số id chỉ gồm một phần tử thì chúng lại trở thành các phép chèn, loại bó và cập nhật trên một quan hệ trong mô hình cơ sở dữ liệu quan hệ [5].

\section{5. ĐẠI SỐ QUAN Hệ TRÊN KHỐI}

Cho $r$ là một khối trên $R=$ (id; $A_{1}, A_{2}, \ldots, A_{n}$ ), ờ đây ta luôn giá thiết rằng $r$ là một khối gồm một tập hũu hạn các phần từ. Cũng tương tự như đại số quan hệ trong mô hình cơ sở dữ liệu quan hệ, ở đây các phép toán của đại số quan hệ lại được áp dụng cho các khối; bên canh đó còn có thêm hai phép toán mới được xây dựng là: tích Đề các theo tập chỉ số và phép nối dài.

Đối vói các phép hợp, giao và trừ thì hai khối tham gia phải là khả hợp (nghĩa là nó cùng một lược đồ khối).

\section{a. Phép hợp:}

Cho 2 khối $r$ và $s$ khả hợp, khi đó hợp của $r$ và $s$, kí hiệu $r \cup s$ là một khối gồm các phần tử thuộc một trong trong 2 khối $r$ và $s$ đã cho.

Biểu diễn hình thức phép hợp có dạng:

$$
r \cup s=\{t \mid t \in r \text { hoặc } t \in s\} \text {. }
$$

b. Phép giao:

Cho 2 khối $r$ và $s$ khả hợp, khi đó giao của 2 khối $r$ và $s$ là một khối, kí hiệu $r \cap s$, mà các phần tử của nó thuộc đồng thời cá 2 khối $r$ và $s$ dã cho.

Biểu diễn hình thức phép giao có dạng:

$$
r \cap s=\{t \mid t \in r \text { và } t \in s\} .
$$

\section{c. Phép trừ:}

Cho 2 khối $r$ và $s$ khả hợp, khi đó hiệu của 2 khối $r$ và $s$ là một khối, kí hiệu $r-s$, mà 
các phần tử của nó thuộc $r$ nhưng không thuộc $s$.

Biểu diễn hình thức phép trừ có dạng:

$$
r-s=\{t \mid t \in r \text { và } t \notin s\} \text {. }
$$

(Ta có mối quan hệ giữa phép giao và phép trừ: $r \cap s=r-(r-s)$ ).

\section{d. Tích Đề các:}

Định nghĩa 1. Cho $R=\left(\right.$ id; $\left.A_{1}, A_{2}, \ldots, A_{n}\right), S=\left(\right.$ id; $\left.B_{1}, B_{2}, \ldots, B_{m}\right)$, ờ đây $\left\{A_{1}, A_{2}, \ldots, A_{n}\right\}$ $\cap\left\{B_{1}, B_{2}, \ldots, B_{m}\right\}=\emptyset$. Khi đó tích Đề các của hai khối $r(R)$ và $s(S)$ là một khối, kí hiệu $r \times s$, khối này có khung $R \times S=\left(\right.$ id; $A_{1}, A_{2}, \ldots, A_{n}, B_{1}, B_{2}, \ldots, B_{m}$ ), mối phần tứ thuộc khối này là một bộ gồm $n+m$ ánh xạ, trong đó $n$ ánh xạ đầu có dạng một phần tử thuộc $r$, còn $m$ ánh xạ sau có dạng một phần tử thuộc $s$.

Biểu diễn hình thức của tích Đề các có dạng:

$$
r \times s=\{t \mid t(R) \in r \text { và } t(S) \in s\},
$$

trong đó $t=\left(t^{1}, t^{2}, \ldots, t^{n}, t^{n+1}, \ldots, t^{n+m}\right), t(R)=\left(t^{1}, t^{2}, \ldots, t^{n}\right)$ và $t(S)=\left(t^{n+1}, \ldots, t^{n+m}\right)$.

\section{e. Tính đề các theo tập chì sô:}

Định nghĩa 2. Cho $R=\left(\mathrm{id} ; A_{1}, A_{2}, \ldots, A_{n}\right), S=\left(\mathrm{id}^{\prime} ; A_{1}, A_{2}, \ldots, A_{n}\right)$. Khi đó tích Đề các của 2 khối $r(R)$ và $s(S)$ theo tập chỉ số là một khối, kí hiệu $r \times \times_{\mathrm{id}} s$, khối này có khung $R \times_{\text {id }} S=\left\{\mathrm{id} \amalg i d^{\prime} ; A_{1}, A_{2}, \ldots, A_{n}\right\}$, với id $\amalg i d^{\prime}$ là kí hiệu tích rời rạc của hai tập chỉ số id và id'. Mối phần tứ thuộc khối này là một bộ gồm $n$ ánh xạ $\left(t^{i}, t^{2}, \ldots, t^{n}\right)$ với $t^{i}: \mathrm{id} \amalg \mathrm{id}^{\prime} \rightarrow A_{i}, i=1, \ldots, n$, mối ánh xạ này được cám sinh từ 2 ánh xạ thứ $i$ tương ứng của $r$ và $s$.

Cụ thể hơn, giá sứ có 2 phần tự là $t_{r} \in r$ và $t_{s} \in s: t_{r}=\left(t_{r}^{1}, t_{r}^{2}, \ldots, t_{r}^{n}\right), t_{s}=\left(t_{s}^{1}, t_{s}^{2}, \ldots, t_{s}^{n}\right)$, khi đó ta có ánh xạ cám sinh của $t_{r}$ và $t_{s}$, phần tứ cám sinh của $t_{r}$ và $t_{s}$ kí hiệu là $t_{r s}$.

Gọi $j_{1}: \mathrm{id} \rightarrow \mathrm{id} \amalg \mathrm{id}^{\prime}, j_{2}: \mathrm{id}^{\prime} \rightarrow \mathrm{id} \amalg i d^{\prime}$ là các phép nhúng thì ta được:

$$
t_{r s} j_{1} \in r \text { và } t_{r s} j_{2} \in s \text {. }
$$

Biểu diễn hình thức của chúng có dạng:

$$
r \mathrm{X}_{\mathrm{id}} s=\left\{t \mid t j_{1} \in r \text { và } t j_{2} \in s\right\} \text {. }
$$

\section{g. Phép chiêu:}

Cho $R=$ (id; $A_{1}, A_{2}, \ldots, A_{n}$ ), $r$ là một khối trên $R$.

Khi đó ta gọi $P=\left(\mathrm{id}^{\prime} ; A_{i_{1}}, A_{i_{2}}, \ldots, A_{i_{h}}\right)$ là lược đồ con của lược đồ $R$ nếu id' $\subseteq \mathrm{id}$, $A_{i_{j}} \in\left\{A_{1}, A_{2}, \ldots, A_{n}\right\}, j=1, \ldots, h$.

Một phép chiếu của khối $r$ trên lược đồ con $P$, kí hiệu $\Pi_{P}(r)$, là một khối có lược đồ khối $P$ và mỗi phần tử thuộc khối này có dạng:

$\left.\left(t^{i_{1}}, t^{i_{2}}, \ldots, t^{i_{h}}\right)\right|_{\mathrm{id}}$, trong đó: $t^{i_{j}} \in\left\{t^{1}, t^{2}, \ldots, t^{n}\right\}, j=1, \ldots, h$, và $\left(t^{1}, t^{2}, \ldots, t^{n}\right) \in r$.

Biểu diễn hình thức của phép chiếu có dạng:

$$
\Pi_{P}(r)=\left\{\left.\left(t^{j_{1}}, t^{j_{2}}, \ldots, t^{j_{h}}\right)\right|_{\mathrm{id}^{\prime}} \mid t^{i_{j}} \in\left\{t^{1}, t^{2}, \ldots, t^{n}\right\}, j=1, \ldots, h,\left(t^{1}, t^{2}, \ldots, t^{n}\right) \in r\right\} .
$$

Ta dễ dàng chứng minh được một số tính chất sau đây của phép chiếu: 
Mệnh đề 5.1 .

1) $\Pi_{P}\left(\Pi_{P}(r)\right)=\Pi_{P}(r)$

2) Nếu $P \subseteq Q$ thi $\Pi_{P}\left(\Pi_{Q}(r)\right)=\Pi_{P}(r)$

3) $\Pi_{P}(r \cup s)=\Pi_{P}(r) \cup \Pi_{P}(s)$

4) $\Pi_{P}(r \cap s)=\Pi_{P}(r) \cap \Pi_{P}(s)$

5) $\Pi_{P}(r-s) \supseteq \Pi_{P}(r)-\Pi_{P}(s)$

ơ đây $r$ và s là các khối khả hợp trên lự̛c đầ $R$, còn $P$ và $Q$ là các lược đồ con của lự̛c đ้̋̀ $R$.

\section{h. Phép chọn:}

Cho $R=\left(\right.$ id; $\left.A_{1}, A_{2}, \ldots, A_{n}\right)$ và khối $r(R)$.

Cho một phép chọn nghĩa là ta xây dựng một tập con các phần tử của khối đã cho thỏa mãn biểu thức $F$ xác định. Biểu thức $F$ được diển tả bằng một tổ hợp Boolean cua các toán hạng, mỗi toán hạng là một phép so sánh đơn giản giữa hai biến là hai giá trị điểm của hai ánh xạ thành phần nào đó, hoặc giữa một biến là giá trị điểm của một ánh xạ thành phần và một hằng.

Các phép so sánh trong $F$ là $<,=,>, \geq, \leq, \neq$, còn các phép toán logic trong $F$ là: $\vee \wedge, \neg$.

Biểu diễn hình thức của phép chọn có dạng:

$$
\sigma_{F}(r)=\{t \in r \mid F(t)\}
$$

trong đó $F(t)$ là giá trị đúng của biều thức Boolean $F$ tại phần tử $t \in r$.

Mệnh đề 5.2. Với r và s là các khối trên luợc đầ $R$ ta có:

1) $\sigma_{F}(r \cup s)=\sigma_{F}(r) \cup \sigma_{F}(s)$

2) $\sigma_{F}(r \cap s)=\sigma_{F}(r) \cap \sigma_{F}(s)$

3) $\sigma_{F}(r-s)=\sigma_{F}(r)-\sigma_{F}(s)$

\section{i. Phép kết nổi:}

Cho $R=\left(\mathrm{id} ; A_{1}, A_{2}, \ldots, A_{n}\right)$ và $S=\left(\mathrm{id} ; B_{1}, B_{2}, \ldots, B_{m}\right)$, cùng với 2 khối $r(R)$ và $s(S)$ tương ưng.

Gọi $T=\left(\mathrm{id} ; C_{1}, C_{2}, \ldots, C_{p}\right)$, trong đó $\left\{C_{1}, C_{2}, \ldots, C_{p}\right\}=\left\{A_{1}, A_{2}, \ldots, A_{n}\right\} \cup\left\{B_{1}, B_{2}, \ldots, B_{m}\right\}$.

Phép kết nối của 2 khối $r$ và $s$, kị hiệu $r \bowtie s$ là khối $t(T)$ định nghĩa như sau:

$$
t(T)=\left\{t \mid \exists t_{r} \in r \text { và } t_{s} \in s \text { sao cho } t(R)=t_{r}, t(S)=t_{s}\right\} .
$$

Phép kết nối này cũng gọi là phép kết nối tự nhiên của hai khối $r(R)$ và $s(S)$, đôi khi sử dụng kí hiệu $r * s$.

Đặc biệt khi các khối $r(R)$ và $s(S)$ có tập chỉ số id trong lược dồ khối cúa chúng chỉ gồm 1 phần tư thì các khối này trớ thành các quan hệ và phép kết nối tự nhiên của hai khối lại trớ thành phép kết nối tự nhiên của hai quan hệ trong mô hình cơ sớ dữ liệu quan hệ $[5]$. đã cho.

Nếu $\left\{A_{1}, A_{2}, \ldots, A_{n}\right\} \cap\left\{B_{1}, B_{2}, \ldots, B_{m}\right\}=\emptyset$ thì $r * s$ trở thành tích Đề các của hai khối

Ta có thễ mớ rộng khái niệm kết nối như sau:

Giá sứ $A_{i_{k}} \in\left\{A_{1}, A_{2}, \ldots, A_{n}\right\}, B_{i_{k}}\left\{B_{1}, B_{2}, \ldots, B_{m}\right\}$, và $\operatorname{dom}\left(A_{i_{k}}=\operatorname{dom} B_{i_{k}}, 1 \leq k \leq h\right.$, (ớ 
đây $A_{i_{k}}$ và $B_{i_{k}}$ không nhất thiết phân biệt). Khi đó kết nối của r và s theo $A_{i_{1}}, A_{i_{2}}, \ldots, A_{i_{h}}$, và $B_{i_{1}}, B_{i_{2}}, \ldots, B_{i_{h}}$ là khối $t(T)$, khối này được định nghĩa như sau:

$$
t(T)=\left\{t \mid \exists t_{r} \in r \text { và } t_{s} \in s \text { sao cho } t(R)=t_{r}, t(S)=t_{s}, t_{r}^{i_{k}}=t_{s}^{i_{k}}, 1 \leq k \leq h\right\},
$$
ơ đây $t_{r}=\left(t_{r}^{1}, t_{r}^{2}, \ldots, t_{r}^{n}\right), t_{s}=\left(t_{s}^{1}, t_{s}^{2}, \ldots, t_{s}^{m}\right)$.

Thay cho kí hiệu $r \bowtie s$ ơ đây ta kí hiệu rõ hơn:

$$
t(T)=r\left[t_{r}^{i_{k}}=t_{a}^{i_{k}}, 1 \leq k \leq h\right] s .
$$

\section{k. Phép nối dài:}

Cho hai khối $r\left(\mathrm{id} ; A_{1}, A_{2}, \ldots, A_{n}\right)$ và $s\left(\mathrm{id}^{\prime} ; A_{1}, A_{2}, \ldots, A_{n}\right)$, ơ đó nếu $\mathrm{id}_{1} \cap \mathrm{id}_{2} \neq \emptyset$ mà ta có với $t \in r$ và $k \in s$ :

$$
\begin{aligned}
& \left.t^{1}\right|_{\mathrm{id}_{1} \cap \mathrm{id}_{2}}=\left.k^{1}\right|_{\mathrm{id}_{1} \cap \mathrm{id}_{2}} \\
& \left.t^{2}\right|_{\mathrm{id}_{1} \cap \mathrm{id}_{2}}=\left.k^{2}\right|_{\mathrm{id}_{1} \cap \mathrm{id}_{2}} \\
& \left.t^{n}\right|_{\mathrm{id}_{1} \cap_{\mathrm{id}}}=\left.k^{n}\right|_{\mathrm{id}_{1} \cap_{\mathrm{id}}}
\end{aligned}
$$

thì khi đó ta xây dựng được một phần tự mới có dạng:

$$
u=\left(u^{1}, u^{2}, \ldots, u^{n}\right) \text { vói } u^{h}:{\text { id } \cup \text { id }^{\prime} \rightarrow A^{h}}^{h}
$$

sao cho: $\left.u^{h}\right|_{\mathrm{id}}=t^{h},\left.u^{h}\right|_{\mathrm{id}}=k^{h}, \forall h=1, \ldots, n$, và kí hiệu: $u^{h}=t^{h} *_{\mathrm{id}} k^{h}, \forall h=1, \ldots, n$.

Nhũng phần tứ $u=\left(u^{1}, u^{2}, \ldots, u^{n}\right)$ này tạo ra một khối mới, được kí hiệu: $r *_{\mathrm{id}} 3$, gọi là khối nối dài của 2 khối $r$ và $s$.

Phép toán được xây dựng ớ trên gọi là phép nối dài của 2 khối $r$ và $s$ đã cho.

Biểu diễn hình thức cưa phép nối dài có dạng:

$$
r *_{\mathrm{id}} s=\left\{u=\left(u^{j}\right)_{j=\overline{1, n}} \mid\left(\left.u^{j}\right|_{\mathrm{id}}\right)_{j=\overline{1, n}} \in r \text { và }\left(\left.u^{j}\right|_{\mathrm{id}}\right)_{j=\overline{1, n}} \in s\right\} .
$$

Mệnh đệ 5.3. Giả sứ $r(R), r^{\prime}(R), q(Q), s(S)$ là các khối đãa cho, khi đó ta có:

1) $(q \bowtie r) \bowtie s=q \triangleright \triangleleft(r \triangleright s)$

2) $\left(r \cup r^{\prime}\right) \bowtie s=(r \bowtie s) \cup\left(r^{\prime} \bowtie s\right)$

3. $\left(r \cap r^{\prime}\right) \bowtie s=(r \bowtie s) \cap\left(r^{\prime} \bowtie s\right)$

4. $\left(r-r^{\prime}\right) \bowtie s=(r \bowtie s)-\left(r^{\prime} \bowtie s\right)$

\section{Phép chia:}

Cho khối $r\left(\right.$ id; $\left.A_{1}, A_{2}, \ldots, A_{n}\right)$ và khối $s\left(\right.$ id; $A_{i_{1}}, A_{i_{2}}, \ldots, A_{i_{h}}$ ), trong đó

$\left.A_{i_{k}} \in\left\{A_{1}, A_{2}, \ldots, A_{n}\right)\right\}, \forall k=\overline{1, h}$, khi đó phép chia của khối $r$ cho khối $s$, kí hiệu $r \div s$, là một khối gồm các phần tư $t=\left(t^{1}, t^{2}, \ldots, t^{n-h}\right)$ sao cho $\forall u=\left(u^{1}, u^{2}, \ldots, u^{h}\right), u \in s$ thì phần từ $t u \in r$, ơ đây phần tử $t u$ có dạng: $t u:=\left(t^{1}, t^{2}, \ldots, t^{n-h}, u^{1}, u^{2}, \ldots, u^{h}\right)$.

Biều diễn hình thức của phép chia có dạng:

$$
r \div s=\{t \mid \forall u \in s, t u \in r\} \text {. }
$$

Trong trường hợp các khối $r$ và $s$ có tập chỉ số id chỉ gồm 1 phần tử thì khi đó các khối này trở thành các quan hệ và phép toán chia của hai khối trớ thành phép toán chia của hai quan hệ trong mô hình cơ sở dữ liệu quan hệ [5]. 
Mệnh đề 5.4. Giả sử $R=\left(i d ; A_{1}, A_{2}, \ldots, A_{n}\right), S=\left(i d ; A_{i_{1}}, A_{i_{3}}, \ldots, A_{i_{h}}\right)$, trong đó $A_{i_{k}} \in$ $\left\{A_{1}, A_{2}, \ldots, A_{n}\right\}, \forall k=\overline{1, h}$ và $r(R), r^{\prime}(R), s(S), s^{\prime}(S)$ là các khối đã cho, khi đó ta có:

1) $\left(r \cup r^{\prime}\right) \div s \supseteq(r \div s) \cup\left(r^{\prime} \div s\right)$.

2) $\left(r \cap r^{\prime}\right) \div s=(r \div s) \cap\left(r^{\prime} \div s\right)$.

3) $\left(r-r^{\prime}\right) \div s \subseteq(r \div s)-\left(r^{\prime} \div s\right)$.

4) $N$ éu $s \subseteq s^{\prime}$ thi $(r \div s) \supseteq\left(r \div s^{\prime}\right)$.

\section{TÀI LIỆU THAM KHẢO}

[1] Armstrong W.W., Dependency structures of database relationships, Proc. 1974 EFIP Congress, pp. 580-583, North-Holland, Amsterdam.

[2] Beeri C., On the membership problem for functional and multivalued dependencies, ACM Trans. on Database System 5 (3) (1980) 241-259.

[3] Demetrovics J., Nguyen Xuan Huy, Closed sets and translation of relation schemes, Computers Math. Applic. 21 (1) (1991) 13-23.

[4] Demetrovics J., Nguyen Xuan Huy, Representation of closure for functional, multivalued and join dependencies, Computers and Artificial Intelligence 11 (2) (1992) 143-154.

[5] Maier D., The Theory of Relational Databases, Computer Science Press, Rockville, Md., 1983.

[6] Ullman J.D., Principles of Database Systems, Computer Science Press, Rockville, Md., 1982.

[7] Ullman J. D., Principles of Database and Knowledge - Base systems, Vol. 1, 2, Computer Science Press, 1989.

(1) Viện Công nghệ thông tin, Trung tâm KHTN và CNQG.

Received: March 10, 1998

(2) Trương Dại học Su phạm - Dại học Quóc gia Hà Nội. 